collect in the pulmonary vesicles, it may of two of the cases detailed, demonstrated, incorporate itself with the substance of clearly, the carbonaceous nature of the the lung, its presence, as a source of irri- deposit, and its unlikeness to any of the tation, may react upon the existing dis- secretions, or to any animal substance ease; the respiratory functions will thus whatever.

become embarrassed, and those organic changes in the substance of the lung ensue, which, after an uncertain period, unfit this organ for its purposes in the animal economy.

That some such predisposition as is here presumed is necessary to the accession of the disease, is proved by its rareness; and as disense of the lungs is comparatively unfrequent among colliers, the rareness of the affection (if the predisposition consists in such disease) stands still further accounted for. Certainly, in the cases detailed, confessedly difficult though it be in such a matter as the present to distinguish betwixt cause and effect, the impression on my mind is, that pulmonary disease, arising from natural and cognizable causes, preceded in every instance the accession of the peculiar affection under consideration.

It may be said that the same objection as was valid against the inhalation of lamp-sooot, as the cause of this disease, may be urged against the inhalation of coal-dust. But it ought to be recollected, that while the former of these causes is identical in its nature in all cases, the existence at all of the latter depends upon a variety of circumstances, as the nature of the coal, the methol of working it, \&c. Thus, when the coal is very sott, and wrought small, as in the English collieries, or when the workings are of a wet or damp nature, from the neighbourhood of springs, or from constant lcakage, the production of this fine powder can scarcely take place. But when, as in the pits in which these cases occurred, the workings are perfectly diy, when the coal, as in them, is close, hard, and compact in its fracture, and when it is wrought large by the continucd and laborious application of the pick, then we have circumstances in all respects favourable to the production of this tine dust.

Were it necessary to advocate still further the opinion here advanced, respecting the origin of this disease, the collateral evidence of the analysis of the melanotic matter in Dr. Gregory's case might be adduced, as well as the direct proof afforded by the fact, that the disease only occurs in colliers, and in artificers who, like them, are exposed to the inhalation of carbonaccons matter; thus a case lately occurred in this vicinity, in the person of a workman employed in dusting moulds for fine castings with finely powdered charcoal. Finaly, analysis of the spitum

The process pursued in this analysis was the subjecting the matter to the action of nitric acid at the hoiling point, subsequently to that of a solution of caustic potass, and, finally, to the operation of heat; and though the aralysis was not carried so far as to ascertain the identity of the matter with coal, yet its affinity to charcoal was sufficiently evident.

Cambuslang, Sept. 12th, 1834.

\title{
HYDROPHOBIA
}

FOLLOWING THE BITE OF A DOG NOT SUPPOSED TO BE MAD.

\section{To the Editor of The Lancers.}

Sir,-The very peculiar manner in which the following case of hydrophobia was excited, renders it, in a professional point of view, one of considerable interest. Being thus inpressed, $I$ feel it a duty to communicate it to you for insertion in your excellent journal.

I am Sir,

Your obedient servant, E. L. Know les, Surgeon. Soham, Cambridgeshire,

Sept. 5th, 1834.

Case.-Wm. Hills, of Soham, what. seven. His mother states, that about five months ago he was bitten by a dog which was exasperated by the continued teazing of this and other boys. IJis shoulder was much lacerated, but the wound was considered of little importance, the animal being well and still in existence. It was simply dressed, and it soon healed; from which period, till Saturday, June the $9 \mathrm{th}$, the boy appeared to be perfectly well; he then complaincd of thirst, nansca, and a disinclination to move. On Sunday his symptoms had much increased, and he expressed very great aversion to being washed, according to usual custom. Notwithstanding these symptoms, his friends were still impressed with the idea of the disorder being a bilious attack, until ironday, at four p.m., when the symptoms be. came so augmented as to induce them to apply for medical assistance.

On entering the room, the first symptom that caught the eye was the great distress depicted in the patient's comte. nance. The eyelids were opened to their fullest extent, accompanied by considera- 
ble dilatation of the pupils; the face, / bite his attendants, who carinot now preneck, and chest, were bathed in perspira- vail upon him, either by threats or retion; the angles of the mouth were drawn / ward, to suffer the smallest quantity of up by the spasmodic action of the zygomatic fluid to pass his lips. From this time he muscles, producing a horrid grin, and he gradnally became worse, and at 12 p.m. was incessantly spitting a frothy mucus. death put a period to his extreme agony. When spoken to, he answered in a hurried REMARKs.-I am sorry to say that no manner, and in broken accents. There post-mortem examination was permitted. was no pain in the site of the wound, but There is, in my opinion, in this case, a considerable uneasiness was experienced character well worthy the attention of the in the region of the heart. Pulse 90, fee- profession generally, as it may unfold the ble, irregular, and intermitting; respira- answer to a question which has long been tion hurried, and deglutition appeared to be doubted by the medical world, viz. wheentirely suspended. Notwithstanding he ther the canine race are capable of com. had great thirst, the sight of the smallest municating hydrophobia under a state of quantity of water produced the most vio- irritation or excitement, when not actually lent spasms and jactitations of the whole labouring under the disease. In Dr. body. The muscles of the back were vio- Thomas's Practice of Physic, the following lently contracted, and the arched appear- remark is noted:-M. Rossi, in the Mem.de ance existed which is so frequently ob- l'Academie de Tuin, tom, vi., endeavours served in tetanus, with which disease it to demonstrate, by cases, that animals, might have been confounded had it not previously healthy, when enraged or irribeen for the effects which the appearance tated, in a high degree, are capable of of fluids produced, and the absence of a re- communicating the disease by their bite, cent wound. The spasms occurred, when although Dr. T. ccnsiders that sufficient not excited, about every five minutes.

At six p.m. On visiting him at this establish the principle satisfactorily. I period, I found him in every respect the made every inquiry, both of the patient same, with the exception of having a great and, since his death, of his mother, as to antipathy to his bed; he was therefore al- whether he was bitten by any other dog, lowed to dress and walk during the in- and they positively answercd in the netervals of the paroxysm. We now suc- gative. What led me to the investigation ceeded, by means of a small reward, in in a more minute degree than $I$ otherwise prevailing upon him to swallow a strong should have pursued it, was the fact that opiate. He made repeated attempts ere the animal which inflicted the wound conhe could accomplish it; and although it tinues alive and well up to the present was succeded every time by a violent time. Therefore the facts of this case are spasmodic constriction, yet, so determined in perfect unison with the sentiments of was his character, that he persisted to the the French author: and as practical knowlast degree in his efforts. The warm-bath ledge is of more value than theoretical and an opiate enema were now proposed, speculation, I shall not trespass upon the in the hope of alleviating the spasmodic valuable time of your numerous readers. action ; but we were obliged to abandon it, by advancing any physiological hypotheses in consequence of the powerful and dis- on the subject.*

tressing effect produced on the patient's mind. The avidity with which he took an orange was astonishing; violent spasms however instantly succeeded, particularly about the throat and adjacent parts. He appeared perfectly sensible, an l repeatedly expressed his thanks for the small reward given for trying his utmost to overcome the difficulty that arose in swallowing. Great anxiety still prevailed in his countenance, with sighing, and every word was succeeded by a conzulsive sob. The draught was ordered to be repeated every hour.

Ten p.m. The malady is evidently adrancing, although the spasms continue much the same. Symptoms of exhaustion are now evident. His friends are unable to confine him in bed, except by the assistance of two men. $\mathrm{He}$ is constantly spitting, and has a great propensity to 944 of the present $\mathrm{No}_{\mathrm{O}}$

No. $57 \%$.

EMPLOYMENT OF

\section{FREE INCISIONS IN GLOSSITIS.}

\section{To the Editor of The Lancet.}

SIR, - In a communication in THE LANCET (page 634) from Mr. Horne on the efficacy of the nitrate of silver in glossitis, there is a case related in which the nitrate was applied every morning for the space of a fortnight, and in the early stage of the disease twice a day. A fortnight is a long time to con-

* An opinion on this subject expressed y Sir B. Brodie, is recorded at page 4 of the present $\mathrm{No}$.
$3 \mathrm{O}$ 